\title{
Las redes de colaboración científica y su efecto en la productividad. Un análisis bibliométrico
}

\author{
Alejandra García Hernández *
}

Artículo recibido:

21 de septiembre de 2012.

Artículo aceptado:

4 de diciembre de 2012.

\section{RESUMEN}

Una gran parte de los estudios bibliométricos sobre colaboración científica muestra que existe un efecto de la colaboración entre investigadores en su productividad, sin embargo poco se conoce sobre la forma en que la colaboración beneficia o inhibe la productividad científica. El análisis de redes sociales provee de herramientas y métodos que permiten analizar la estructura de las redes de colaboración y también existen algunos estudios bibliométricos de redes de co-autoría en publicaciones que utilizan métodos de análisis de redes, pero aunque la mayoría de estos estudios se han realizado a nivel de disciplina científica no se ha identificado evidencia empírica que analice las redes de co-autoría y su relación con la productividad científica a nivel de grupo de investigación académico. En

* Centro de Investigación en Matemáticas, CIMAT, México. algarcia@cimat.mx

INVESTIGACIÓN BIBLIOTECOLÓGICA, Vol. 27, Núm. 59, enero/abril, 2013, México, ISSN: 0187-358X. pp. 159-175 
este artículo se analizan las redes de co-autoría de 76 grupos de investigación de la Universidad Politécnica de Valencia, y el efecto que tiene la estructura de la red de los grupos en su productividad científica. Los resultados muestran que existe una relación entre las variables de red y la productividad de los grupos. La centralidad de la red y el tamaño de la red del grupo son dos importantes variables de red para la productividad científica de los grupos. La densidad de la red y la presencia de agujeros estructurales no mostraron un efecto significativo en la productividad a nivel de grupo de investigación.

Palabras clave: Redes de co-autoría; Análisis de redes sociales; Productividad científica; Grupos de investigación.

\section{ABSTRACT}

A bibliometric analysis of scientific collaboration networks and their effect on productivity Alejandra García-Hernández

Bibliometric studies of scientific collaboration show a relationship between collaboration and scientists' productivity; however, little is known about how collaboration might inhibit or enhance scientific productivity. Social Network Analysis (SNA) provides tools and methods for analyzing the structure of collaboration networks. Some bibliometric studies of co-authorship networks are applying network techniques to study scientific networks within certain disciplines. This paper is focused on the relationship between co-authorship networks and productivity of research groups. The study samples 76 academic research groups of the Polytechnic University of Valencia. The principal results show that there is a relationship between network variables and a given group's productivity, demonstrating that network centralization and size are important variables explaining variations of a group's productivity. Moreover, network density and structural gaps in a network had no effect on a group's productivity.

Keywords: Co-authorship networks; Social network analysis; Scientific productivity; Research groups. 


\section{INTRODUCCIÓN}

T a colaboración científica ha sido ampliamente estudiada a través de análisis bibliométricos, algunos de los cuales utilizan las publicaciones científicas para extraer información referente a la colaboración entre investigadores, instituciones o países, con la finalidad de conocer el impacto que tiene la colaboración en los resultados de investigación. Estudios recientes están enfocando sus esfuerzos en analizar la colaboración científica utilizando herramientas de análisis de las redes sociales (SNA) (Abbasi y Altmann, 2010).

$\mathrm{El}$ análisis de redes sociales ha sido ampliamente utilizado en áreas que tienen que ver con la administración, la sociología y la psicología, áreas en las que existe un gran cuerpo de literatura que relaciona las propiedades de las redes sociales con la productividad a diferentes niveles organizacionales, este tipo de estudios por lo general se basa en teorías provenientes del capital social (Luo, 2005; Oh et al., 2006), y se enfocan en identificar el tipo de estructuras de red que inhiben o mejoran la productividad organizacional

El análisis de redes sociales provee de un gran número de métodos que ayudan a revelar patrones de relaciones interpersonales. Desde la perspectiva del capital social, para Burt (2001) la principal metáfora que surge de los estudios de redes sociales es que "los actores que lo hacen mejor están de cierta manera mejor conectados en la red"; sin embargo el significado de estar mejor conectado todavía no es claro; existe un debate en la literatura científica sobre el efecto que algunas propiedades de la estructura de la red tiene en la productividad de las organizaciones (Reagans y Zuckerman, 2001). Entre las propiedades de las redes que han sido más analizadas en los estudios de administración o que siguen la perspectiva del capital social, se encuentran la centralidad de la red, la densidad de ésta, su tamaño y la presencia de agujeros estructurales en ellas.

La centralidad de la red es la propiedad que identifica a los nodos con mayor número de vínculos dentro de una red (Wasserman y Faust, 1994). Los actores más centrales pueden tener una posición de ventaja respecto a los demás en tanto que tienen más alternativas para satisfacer sus necesidades, más posibilidades de acceso a recursos y se considera que son menos dependientes (Hanneman y Riddle, 2005). A los nodos más centrales en la estructura de una red también se les denomina nodos "estrella" ya que por su alto grado de relación sobresalen de los demás. A nivel grupal la propiedad de centralidad de la red se basa en los índices de centralidad de cada uno de los nodos y se usa para medir el grado en el que la red del grupo está concentrada alrededor de uno o pocos nodos. Luo relaciona la centralidad de la red del grupo con aspectos del liderazgo al considerar que una red centralizada 
puede presentar un liderazgo fuerte, y que es posible que exista mayor control de las actividades del grupo y mayor organización, aunque también una red muy centralizada puede limitar la libertad de acción de los integrantes del grupo (Luo, 2005).

La densidad de la red es la propiedad que mide la proporción de las relaciones presentes en ella sobre el máximo número de relaciones que pueden existir. El número máximo de relaciones está determinado por el número total de nodos que hay en la red (Wasserman y Faust, 1994). La densidad de la red es minimizada cuando no existen relaciones entre los actores y es maximizada cuando todos los actores están relacionados entre sí (Reagans y Zuckerman, 2001).

El tamaño de la red es la propiedad que mide el número de actores o individuos que participan en la red social (Tichy et al., 1979). Cuanto mayor sea el número de contactos de un nodo, mayor será su red y los recursos, la información o el conocimiento a los que tiene acceso.

Los agujeros estructurales son la propiedad de la red que mide la ausencia de relación entre los contactos directos de un nodo; a la ausencia de relación entre dos contactos se le denomina "agujero estructural". Esto no quiere decir que los contactos o los grupos desconectados no sean conscientes de su existencia en la red, sino que simplemente cada uno trabaja en sus actividades y no mantienen un canal de comunicación entre sí. El nodo que tiene contactos con agujeros estructurales entre sí se puede beneficiar dado que tiene acceso a los diferentes flujos de información de cada contacto. Al tener acceso a diferente información el nodo puede beneficiarse de esta oportunidad controlando el flujo de la información e intermediando entre sus diferentes contactos (Burt, 2001).

En estudios recientes de colaboración científica a través de redes de coautoría también están siendo aplicados los métodos del análisis de redes, y se están considerando las teorías o argumentos provenientes del capital social para analizar las redes de colaboración (Perianes-Rodríguez et al., 2010). Los principales resultados han identificado que los investigadores más productivos en su disciplina científica ocupan una posición central en la red y son muy activos en la red de colaboración de su disciplina (Hou et al., 2006; Leij y Goyal, 2006). De acuerdo con Leij y Goyal (2006) la red central de la disciplina está formada sólo por un pequeño grupo de investigadores muy productivos, mientras que la mayoría de los investigadores con baja productividad ocupa una posición periférica en la red. Los investigadores centrales también denominados investigadores "estrella" tienen más de 50 vínculos con otros investigadores, mientras que la mayoría de los investigadores de la disciplina sólo tiene uno o dos vínculos. Newman (2004) también encontró 
algo similar a los resultados de Leij y Goyal en tres disciplinas científicas diferentes, e identificó que los investigadores con muchos vínculos en la red tienden a colaborar con otros investigadores que tienen también redes grandes. También se ha averiguado que la existencia de vínculos entre diferentes departamentos mejora la productividad de los investigadores (Hill, 2008), y a nivel individual Wairepo encontró que la red de un investigador caracterizada por la presencia de agujeros estructurales era la estructura de red que tenía una relación positiva con la productividad de los investigadores (Wairepo, 2006).

Los estudios anteriores muestran que existe una relación entre las propiedades de las redes de co-autoría con la productividad científica de los investigadores o de las organizaciones de investigación. Se observa que la mayor parte de los estudios que han analizado las redes de colaboración científica utilizando co-autoría de publicaciones se han realizado a nivel de disciplina científica (Newman, 2004; Schummer, 2004; Larsen, 2008) o a nivel individual (Lowrie y McKnight, 2004; Hou et al., 2006); el nivel organización como unidad de análisis en el estudio de redes de co-autoría y productividad ha sido poco explorado, aunque existen algunas excepciones como el trabajo de Yousefi-Nooraie et al., aunque ellos sólo consideraron una muestra pequeña de tres centros de investigación (Yousefi-Nooraie et al., 2008), y el trabajo de Hill (2008), quienes estudian las relaciones internas y externas de un departamento de una universidad.

A pesar de la importancia que tiene el grupo de investigación en los estudios de la productividad científica, por ser el contexto más próximo a los investigadores en donde la investigación se lleva a cabo (Rey-Rocha et al., 2002; Cummings y Cross, 2003; Tunzelmann et al., 2003; Cross et al., 2008), son muy pocos los estudios sobre colaboración científica que consideran como unidad de análisis el grupo; la principal razón es la falta de información que existe a este nivel de análisis y la dificultad que hay para obtenerla (VanRaan, 2003; Carayol y Matt, 2004). No se ha identificado algún estudio que analice las redes de co-autoría de los grupos de investigación académicos y el efecto que tienen éstas en la productividad científica de los grupos.

Este trabajo de investigación tiene el objetivo principal de extender el estudio bibliométrico de las redes de co-autoría en publicaciones a nivel de grupo de investigación, así como de analizar el efecto que tiene la estructura de la red en la productividad científica de los grupos; se asume que los investigadores desarrollan estrategias al momento de seleccionar a sus colaboradores (Lowrie y McKnight, 2004; Wairepo, 2006), y son sus estrategias las que van formando la red de colaboración de su grupo de investigación (red de co-autoría). También se realiza un análisis de la relación que existe entre 
la centralidad, la densidad, el tamaño de la red y la presencia de agujeros estructurales en las redes de los grupos de investigación con la productividad científica de sus resultados.

Debido a que estudios recientes consideran que para analizar las redes sociales a nivel de grupo es importante considerar las relaciones que existen entre los integrantes del grupo (relaciones internas) y también las relaciones que el grupo tiene con contactos externos (relaciones externas), ya que ambas pueden tener implicaciones importantes en la productividad del grupo (Ancona, 1990; Oh et al., 2006), en este trabajo se analizan las relaciones internas (densidad y centralidad), y las relaciones externas del grupo (tamaño de la red y agujeros estructurales).

\section{Metodología}

Para identificar la relación que existe entre las propiedades de la red (densidad, centralidad, tamaño, agujeros estructurales) y la productividad de los grupos de investigación académicos, la recolección de los datos se realizó en la Universidad Politécnica de Valencia (UPV). La UPV cuenta con un portafolio tecnológico llamado Sistema CARTA, el cual permitió identificar el total de grupos de investigación que tiene la UPV, el tamaño del grupo, el nombre de sus integrantes y la productividad de los grupos.

Para seleccionar los grupos de la muestra consideramos algunos criterios de selección importantes basándonos en la literatura científica. Se seleccionaron los grupos que trabajan en las ciencias aplicadas y en las ciencias básicas; los grupos que trabajan en ciencias sociales y en humanidades fueron excluidos de la muestra porque algunas investigaciones han demostrado que siguen patrones de colaboración diferentes debido a factores propios de su disciplina científica (Carayol, 2005; CSIC, 2008). Se seleccionaron grupos con al menos 4 años de actividad en tanto que es posible que los grupos de reciente creación puedan estar en desventaja simplemente por su edad. Se seleccionaron sólo los grupos que tenían producción científica en revistas de impacto (revistas indexadas en el ISI), primero porque en este artículo se define la red de colaboración de cada grupo con base en la co-autoría de las publicaciones del grupo, y segundo porque las revistas indexadas en el ISI son consideradas como los principales instrumentos de evaluación sobre la cantidad y la calidad de la investigación; además son utilizadas cada vez con mayor frecuencia para monitorear a las instituciones de investigación y gobiernan las prácticas de publicación de los investigadores de diferentes áreas científicas y países (Paasi, 2005). Se seleccionaron grupos que mostraron un nivel mínimo de 
colaboración y al igual que Oh et al. (2004) se seleccionaron grupos que contaban con al menos tres relaciones de colaboración entre sus integrantes. Finalmente el periodo analizado es de cinco años (2001-2005), pues para poder evaluar resultados científicos se requiere contar con una ventana de datos mínima de cinco años (Jauch y Glueckt, 1975; Newman, 2001).

Para elegir sólo los grupos que tenían producción científica en revistas ISI, primero se realizó la búsqueda de las publicaciones ISI de la UPV durante todo el periodo de análisis 2001-2005. Los criterios de búsqueda en la web del ISI fueron los siguientes:

- Dirección (AD): "UPV" or "Polithe*Univ* Of Valencia" or "Univ*Polit *de Valencia*"or "CSIC-UPV".

- Ciudad (CI): "Valencia" or "Gandía".

- País (CU): "Spain" or "España”.

- Periodo de tiempo (Time span): 2001-2005.

Se integró toda la información en una base de datos y se trabajó conjuntamente con las bases de datos de la UPV. De esta manera se pudo obtener una base de datos completa sobre la producción científica de los grupos de la UPV.

El total de grupos de investigación registrados en el Sistema CARTA hasta el año 2005 fue de 372 grupos. Para obtener la red de colaboración de los grupos se utilizó la co-autoría de las publicaciones de cada grupo y se consideró que existe una relación si dos investigadores son autores en un mismo artículo. Identificamos la red de co-autoría para cada grupo utilizando el software UCINET (Borgatti et al., 2002). Se analizó cada una de las redes y se identificaron los grupos que tenían un mínimo de colaboración de tres relaciones entre sus integrantes, para lo cual se consideraron todos los criterios de selección mencionados anteriormente; la muestra final fue de 76 grupos de investigación y desarrollo.

Para analizar la relación entre las variables de la red de los grupos y la variable de productividad científica se aplicó el análisis de regresión múltiple. El modelo de regresión consta de cuatro variables de red independientes: centralidad de la red, densidad, tamaño de la red externa y agujeros estructurales; una variable dependiente, la productividad científica de los grupos, y tres variables de control: la edad del grupo, la disciplina científica y el tamaño del grupo. 
Para medir las variables de red de cada uno de los grupos se utilizó el Software UCINET el cual fue diseñado para estos propósitos.

- Centralidad: la centralidad de la red de un grupo mide el grado en que las relaciones dentro del grupo están concentradas en uno, varios o en pocos investigadores. Para medir la centralidad de la red se aplicó la medida de centralidad de grado propuesta por Freeman y utilizada en varios estudios (Freeman, 1978; Gaete y Vásquez, 2008; Wong, 2008):

$$
C_{D}=\frac{\sum_{i=1}^{n}\left[C_{D}\left(P^{*}\right)-C_{D}\left(P_{i}\right)\right]}{n^{2}-3 n+2}
$$

En donde $C_{D}$ es el grado de centralidad de la red del grupo, $n$ es el número de integrantes de la red del grupo, $C_{D}\left(P^{*}\right)$ es el grado de centralidad del actor que tiene el valor más alto de centralidad en la red, y $C_{D}(P i)$ es el grado de centralidad del actor $i$.

- Densidad: la densidad de la red de cada grupo varía dentro de un rango de 0 a 1 , en donde un valor de cero significa que no existe relación entre los integrantes del grupo, mientras que un valor de uno significa que todos los integrantes del grupo están relacionados entre ellos y es el nivel máximo de relación que puede haber entre los integrantes del grupo (Wasserman y Faust, 1994). La ecuación general para medir la densidad de cada grupo es la siguiente:

$$
\Delta=\frac{2 L}{g(g-1)}
$$

En donde $\Delta$ es la densidad de la red del grupo, $L$ es el número de relaciones que existen entre los integrantes del grupo y $g$ es el número de actores que forman parte de la red interna del grupo.

- Tamaño de la red: se refiere al número de contactos directos con los que cada uno de los grupos tiene relación. El tamaño de la red del grupo se midió considerando el número de colaboradores que participan en la red externa del grupo (Tichy et al., 1979; Burt, 1983). A través del software UCINET fue posible visualizar el número de investigadores que no pertenecían al grupo pero que sí tenían una relación directa con él. 
- Agujeros estructurales: para medir la presencia de agujeros estructurales en la red externa de los grupos se siguió el procedimiento utilizado por Borgatti para tales propósitos (Borgatti, 1997):

$$
\begin{aligned}
& \text { Redundancia }=2 \mathrm{t} / \mathrm{n} \\
& \text { Tamaño Efectivo }=\mathrm{n}-2 \mathrm{t} / \mathrm{n} \\
& \text { Eficiencia }=(\text { Tamaño Efectivo } / \mathrm{n}) * 100
\end{aligned}
$$

En donde $t$ es el número de relaciones que existen entre los colaboradores externos de cada grupo, y $n$ es el número de colaboradores externos que tiene cada grupo. Se considera que una red eficiente es una red rica en agujeros estructurales, los valores de eficiencia se encuentran dentro del rango de 0 al 100, un valor de 0 es una red sin agujeros estructurales entre los contactos, y un valor de 100 en eficiencia es una red rica en agujeros estructurales entre los contactos externos del grupo.

- Productividad cientifica: la productividad científica se midió tomando como referencia el indicador de publicaciones ISIM; algunos investigadores encontraron que la cantidad de artículos en revistas de calidad es una medida objetiva para la productividad científica (Jauch y Glueckt, 1975). Además el número de artículos en revistas de calidad es el criterio de evaluación de investigación más deseado por los investigadores (Walford, 2000). Cuando se considera cierto nivel organizacional, como en este caso el grupo de investigación, es posible que el tamaño del grupo influya en la cantidad de la producción de artículos (Tunzelmann et al., 2003), en estos casos es recomendable utilizar como indicador de productividad el número de artículos por investigador equivalente a tiempo completo (TIEQ); de esta manera se pueden identificar los grupos de investigación con niveles altos o bajos de productividad con respecto a su capacidad de investigación (Rousseau, 1998):

$$
\text { Productividad }_{\mathrm{G}}=\frac{\text { Número de publicaciones } \text { ISI }_{\mathrm{G}}}{\text { TIEQ }_{\mathrm{G}}}
$$

Donde $G$ es el grupo que se está analizando y TIEQ es el número de investigadores de tiempo completo.

$$
\text { TIEQ }_{G}=\text { Investigadores }_{G}+0.35 \mathrm{PDI}_{\mathrm{G}}
$$


Donde PDI es el personal docente e investigador. Para estimar el personal de investigación de tiempo completo se consideró que el personal docente e investigador dedica el $35 \%$ de su tiempo a actividades de investigación (Azagra-Caro, 2004; Bermeo, 2007).

- Variables de control: para poder analizar la relación entre las variables de red y la productividad científica de los grupos de investigación es necesario controlar el efecto de 3 variables que podrían influir en los resultados, el tamaño del grupo, la edad del grupo y la disciplina científica del grupo (Leenders et al., 2003).

\section{Resultados}

Antes de llevar a cabo el análisis de regresión se realizó la matriz de correlación entre todas las variables a ser consideradas en el modelo: productividad científica (variable dependiente), centralidad, densidad, tamaño de la red externa, agujeros estructurales (variables independientes), tamaño del grupo, edad del grupo y disciplina científica (variables de control). La matriz de correlación se muestra en la Tabla 1.

Tabla 1. Matriz de correlaciones

\begin{tabular}{|l|rrrrrrrr|}
\hline & X1 & X2 & X3 & X4 & X5 & X6 & X7 & X8 \\
\hline X1 Productividad & 1 & & & & & & & \\
X2 Densidad & -0.04 & 1 & & & & & & \\
X3 Centralidad & $0.35^{\star \star}$ & $-0.29^{\star}$ & 1 & & & & & \\
X4 Tamaño de red & $0.34^{\star \star}$ & $-0.29^{\star}$ & $0.25^{\star}$ & 1 & & & & \\
X5 Agujeros estructurales & $0.26^{\star}$ & $-0.42^{\star \star}$ & 0.17 & $0.38^{\star \star}$ & 1 & & & \\
X6 Tamaño de grupo & $-0.35^{\star *}$ & $-0.36^{\star *}$ & -0.05 & $0.37^{\star *}$ & 0.17 & 1 & & \\
X7 Edad de grupo & -0.01 & 0.02 & $-0.22^{*}$ & -0.01 & -0.04 & -0.02 & 1 & \\
X8 Área Científica & -0.17 & 0.07 & -0.08 & 0.21 & 0.06 & $0.23^{\star}$ & 0.08 & 1 \\
\hline
\end{tabular}

** La correlación es significativa al nivel 0,01 (bilateral).

* La correlación es significativa al nivel 0,05 (bilateral).

En la Tabla 1 se puede observar que las variables que tienen una correlación significativa con la productividad científica de los grupos es la centralidad de la red $\left(0.35^{* *}\right)$, el tamaño de la red externa del grupo $\left(0.34^{* *}\right)$, los agujeros estructurales $\left(0.26^{*}\right)$ y el tamaño del grupo $\left(-0.35^{* *}\right)$. El tamaño del grupo es la única variable de control que mostró una relación significativa con la productividad científica, y se observa además que la relación entre ambas es negativa, es decir, que un incremento en la productividad está asociado 
con una disminución en el tamaño del grupo; en este caso parece ser que los grupos pequeños son más productivos que los grupos grandes.

El análisis de correlación también muestra las interrelaciones entre las variables independientes. La centralidad y la densidad muestran una relación negativa $\left(-0.29^{*}\right)$, lo que significa que un incremento en la centralidad de la red del grupo está asociado con una disminución de la densidad de la red, esto tiene sentido si consideramos que el nivel máximo de centralidad de la red se caracteriza por una red en forma de estrella, donde las relaciones se concentran en un solo actor y sus colaboradores no mantienen relación entre ellos, esta no-relación entre los colaboradores hace que el nivel de densidad de la red sea bajo. Se observa también en la matriz de correlaciones que el tamaño de la red externa muestra una relación negativa con la densidad de la red (-0.29*), un incremento en el número de contactos externos del grupo está asociado a una disminución en las relaciones entre los integrantes del grupo; en cambio el tamaño de la red externa muestra una relación positiva con la centralidad $\left(0.25^{*}\right)$, un incremento en la centralidad de la red del grupo está asociado con un incremento en el número de contactos externos del grupo (tamaño de la red externa). Los agujeros estructurales tienen una relación negativa con la densidad de la red $(-0.42 * *)$ y una relación positiva con el tamaño de la red $\left(0.38^{* * *}\right)$, esto último tiene sentido puesto que si se incrementa el número de colaboradores del grupo es posible que no todos se conozcan y estén relacionados, lo que da lugar a la presencia de agujeros estructurales entre los colaboradores.

El tamaño del grupo presenta una relación negativa con la densidad de la red $\left(-0.36^{* *}\right)$, y una relación positiva con el tamaño de la red externa $\left(0.37^{* *}\right)$, lo que significa que entre más grandes sean los grupos las posibilidades de que exista relación entre todos sus integrantes disminuyen, los investigadores se relacionan sólo con algunos investigadores de su grupo. Los resultados muestran también que un incremento en el tamaño del grupo está asociado con un incremento en el número de contactos del grupo, posiblemente porque en grupos más grandes existen mayores posibilidades de que sus integrantes colaboren con diferentes investigadores externos.

Después de analizar la matriz de correlaciones se realizó el análisis de regresión lineal múltiple cuyos resultados se muestran en la Tabla 2 (página siguiente).

En la Tabla 2 se muestra el resumen del modelo, es importante señalar que el modelo sólo explica el $42 \%$ de la variabilidad observada en la productividad científica de los grupos de investigación académicos analizados. Las variables que influyen en la variabilidad de la productividad científica de los grupos, en tanto que muestran un efecto significativo, son la centralidad de la red interna del grupo aunque con un nivel de significatividad bajo (0.49*), 
Tabla 2. Modelo de Regresión para la Productividad Científica

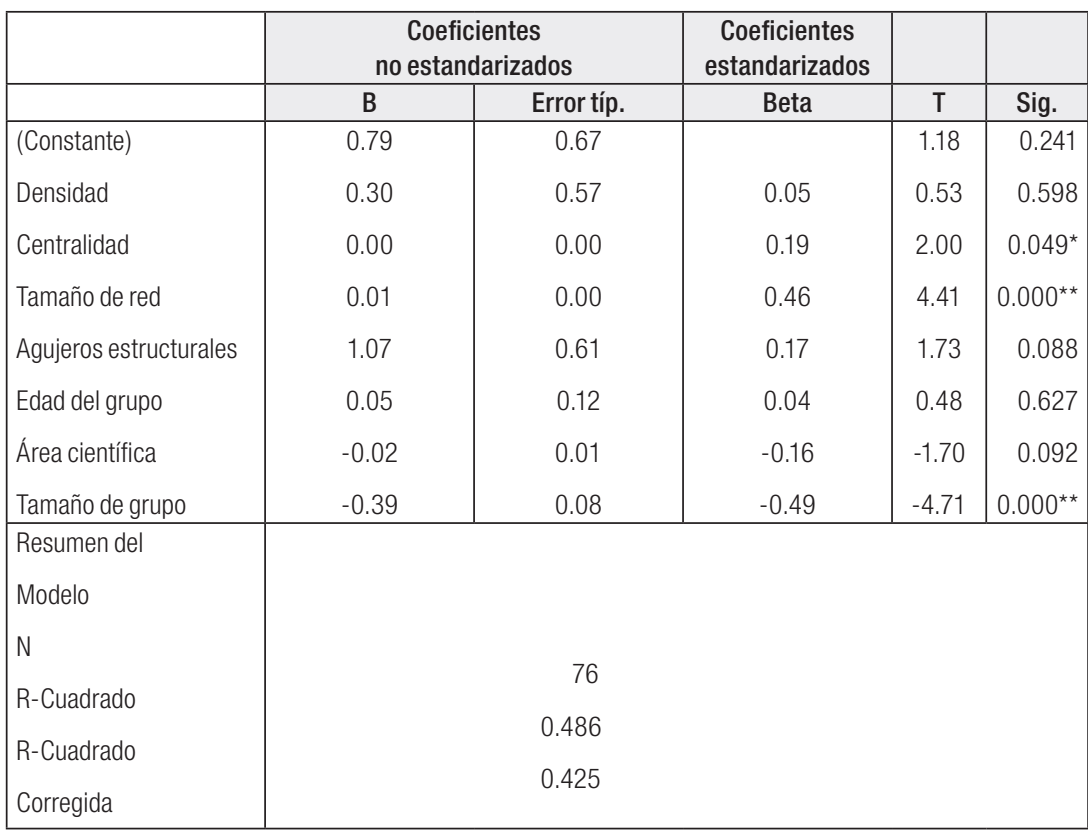

Variable dependiente: productividadcientífica

$* \mathrm{p}<0.05 * * \mathrm{p}<0.01$

el tamaño de la red externa del grupo con un nivel de significatividad alto $(0.000 * *)$ y el tamaño del grupo también con un nivel de significatividad alto $\left(0.000^{* *}\right)$ con la productividad científica. Un incremento en la centralidad de la red interna del grupo está asociado con un incremento en su productividad científica, lo que significa que la concentración de las relaciones del grupo en uno o algunos de sus integrantes favorece su productividad. Un incremento en la red externa del grupo también está asociado con un incremento en su productividad científica, pues un incremento en el número de contactos con los que el grupo tiene relación mejora su productividad. Los resultados muestran también que la densidad de la red y la presencia de los agujeros estructurales no tienen una relación significativa con la productividad científica. Como ya se había mencionado parece ser que para la productividad de los grupos la centralidad es más importante que el grado de interacción entre los integrantes del grupo (densidad) a nivel de red interna, mientras que a nivel de red externa, según los resultados del análisis de regresión (Tabla 2), es más importante para la productividad científica de los grupos la cantidad de contactos con los que el grupo mantiene una relación, que la presencia o ausencia de relación entre sus contactos (agujeros estructurales). 


\section{DisCUSIÓN Y CONCLUSIONES}

Los resultados de esta investigación muestran que a nivel interno del grupo, la centralidad de la red tiene un efecto significativo en su productividad, aunque de manera débil. Lo anterior coincide con los argumentos propuestos por Luo (2005), quien menciona que para la productividad es necesario cierto nivel de centralidad o liderazgo en los grupos ya que de esta manera los grupos tienen mejor organización y control sobre las actividades que realizan. Este resultado es importante ya que demuestra que concentrar las relaciones de un grupo en uno o unos pocos investigadores "centrales" beneficia su productividad, tal y como lo sugieren los resultados de Yousefi-Nooraie et al. (2008) a nivel de centro de investigación, y los resultados de Leij y Goyal (2006) a nivel de disciplina científica; estos últimos identificaron a nivel de disciplina una red con forma de estrella integrada por unos pocos investigadores centrales; los resultados de este artículo extrapolan este fenómeno a nivel de grupo de investigación. A nivel individual algunos estudios también han demostrado que los investigadores más centrales en la red de su disciplina se caracterizan por ser muy productivos (Hou et al., 2006; Leij y Goyal, 2006).

El efecto significativo de la centralidad de la red en la productividad científica se ha observado a diferentes niveles de observación, a nivel de grupo de investigación es posible que la presencia de investigadores "estrella" beneficie la dirección y la organización de sus actividades y los procesos necesarios para la creación del conocimiento científico; de esta manera las actividades en los grupos se realizan de manera más efectiva ya que los investigadores "estrella" poseen la experiencia y el conocimiento para la rápida solución de problemas (Leij y Goyal, 2006; Lowrie y McKnight, 2004; Yousefi-Nooraie et al., 2008).

En cuanto a la red externa del grupo se identificó que sólo la cantidad de colaboradores del grupo tiene un efecto significativo en su productividad y que, a diferencia de lo que se esperaba, la presencia de agujeros estructurales no muestra un efecto significativo. Estos resultados coinciden con los argumentos de Ancona (1990) y Oh et al. (2006) en que las relaciones del grupo con contactos externos son importantes para su productividad; y con los resultados de Hill (2008) quien a nivel de departamento universitario encontró que las relaciones del departamento con otros departamentos de la universidad mejoraba su productividad. También coinciden con los resultados a nivel de investigador de Hou et al. (2006) y de Leij y Goyal (2006), ya que identificaron que los investigadores más productivos en su disciplina científica también son muy activos en su red; es decir, que tienen relación 
con muchos investigadores de su disciplina. Como se mencionó anteriormente podría ser que la presencia de agujeros estructurales en la red externa del grupo no muestre un efecto significativo en la productividad, debido a la correlación positiva que existe entre el tamaño de la red externa del grupo (número de colaboradores) y la presencia de agujeros estructurales, es posible que los beneficios de los agujeros estructurales en la productividad científica de los grupos están ya explicados por la variable del tamaño de la red externa del grupo.

Con esta investigación se confirma que los estudios bibliométricos de análisis de redes de co-autoría en publicaciones científicas ayudan a identificar y analizar los patrones de colaboración de los grupos de investigación, y el efecto de las redes en los resultados científicos de los grupos. Existen algunas propiedades de las redes de los grupos que sí muestran un efecto significativo en la productividad científica mientras que otras no, es importante identificar el efecto de cada una de ellas para poder diseñar estrategias de colaboración científica más efectivas. Además se pudo comprobar con esta investigación que cuando se quiere estudiar la estructura de red de co-autoría de los grupos de investigación es importante también analizar tanto la estructura de red interna del grupo como su red externa, ya que ambas tienen implicaciones importantes en su productividad.

Por último es importante considerar algunas limitaciones de esta investigación: se analizó sólo la estructura de red a través de co-autoría en publicaciones, y existen relaciones entre investigadores que no son visibles a través de la co-autoría, es posible extender esta investigación utilizando otro tipo de documentos, entrevistas o encuestas realizadas con los investigadores. En este estudio sólo se consideró como resultado de investigación la productividad científica de los grupos, sería interesante considerar otro tipo de resultados de investigación y analizar ahí el efecto que tiene la estructura de la red. Sólo se analizó el contexto del grupo de investigación académico de una universidad pública pero se puede extender esta investigación a grupos que se encuentran en otro tipo de contexto.

\section{REFERENCIAS}

Abbasi, A. y Altmann, J. (2010), A Social Network System for Analyzing Publication Activities of Researchers, TEMEP Discussion Paper, South-Korea, Seoul National University.

Ancona, D. G. (1990), "Outward bound: Strategies for team survival in an organization", en Academy of Management Journal, 33(2): 334-365. 
Azagra-Caro, J. (2004), La contribución de las universidades a la innovación: efectos del fomento de la interacción universidad-empresa y las patentes universitarias, Departamento de Análisis Económico, Valencia, Universidad de Valencia, Tesis doctoral.

Bermeo, A. H. (2007), Rendimiento y colaboración cientifica en la investigación académica. Estudio del caso de los grupos de investigación de la Universidad Politécnica de Valencia, Departamento de Proyectos de Ingeniería, Valencia, Universidad Politécnica de Valencia, Tesis doctoral.

Borgatti, S. P. (1997), "Structural holes: Unpacking Burt's redundancy measures", en Connections, 20(1): 35-38.

Borgatti, S. P., Everett, M. G. et al. (2002), Ucinet for Windows: software for social network analysis, Harvard: Analytic Technologies.

Burt, R. S. (1983), "Range", capítulo 9 de Applied network analysis: A methodological introduction, Burt and Minor (eds.).

(2001), "Structural holes versus network closure as social capital", en N. Lin, K. Cook y R. S. Burt, Social capital: Theory and research, New York, Aldine de Gruyter.

Carayol, N. (2005), An economic theory of academic competition: Dynamic incentives and endogenous cumulative advantages, Mimeo, BETA, University Louis Pasteur, Strasbourg.

Carayol, N. y Matt, M. (2004), "Does research organization influence academic production? Laboratory level evidence from a large European university", en Research Policy, 33, 1081-1102.

Cross, R.; Ehrlich, K. et al. (2008), "Managing collaboration: Improving team effectiveness through a network perspective", en California Management Review, 50(4).

CSIC (2008), Impacto de la producción científica de la Comunidad Valenciana, Comunidad Valenciana, Consejo Superior de Investigaciones Científicas-CSIC.

Cummings, J. N. y Cross, R. (2003), "Structural properties of work groups and their consequences for performance", en Social Networks, 25: 197-210.

Freeman, L. C. (1978), "Centrality in social networks conceptual clarification", en Social Networks, 1: 215-239.

Gaete, J. M. y Vásquez, J. I. (2008), "Conocimiento y estructura en la investigación académica: una aproximación desde el análisis de redes sociales", en REDES-Revista hispana para el análisis de redes sociales, $14(5)$.

Gulbrandsen, M. (2000), "Research quality and organizational factors: an investigation of the relationship", en Norwegian University of Science and Technology (NTNU).

Hanneman, R. A. y Riddle, M. (2005), Introduction to social network methods, Riverside, CA, University of California, Riverside (disponible en http://faculty.ucr.edu/ hanneman).

Hill, V. A. (2008), Collaboration in an Academic Setting: Does the Network Structure Matter?, CMU-ISR-8-128, Carnegie Mellon University. 
Hou, H.; Kretschmer, H. et al. (2006), "The structure of scientific collaboration networks in Scientometrics", en Scientometrics, 75(2): 189-202.

Jauch, L. R. y Glueckt, W. F. (1975), "Evaluation of university professors' research performance", en Management Science, 22 (1): 66-75.

Larsen, K. (2008), "Knowledge network hubs and measures of research impact, science structure, and publication output in nanostructured solar cell research", en Scientometrics, 74(1): 123-142.

Leenders, R., Engelen, J. et al. (2003), "Virtuality, communication, and new product team creativity: A social network perspective", en Journal of Engineering and Technology Management, 20(1-2): 69-92.

Leij, M. y Goyal, S. (2006), Strong ties in a small world, Tinbergen Institute Discussion Paper.

Lowrie, A. y McKnight, P. J. (2004), "Academic research networks: A key to enhancing scholarly standing", en European Management Journal, 22(4): 345-360.

Luo, J. D. (2005), "Social network structure and performance of improvement teams", en Int. J., Business Performance Management, 7(2): 208-223.

Newman, M. E. J. (2001), "Scientific collaboration networks. II. Shortest paths, weighted networks, and centrality", en Physical Review E, 64.

(2004), "Coauthorship networks and patterns of scientific collaboration", en Proc. Natl. Acad. Sci., 101:5200-5205.

Oh, H.; Chung, M. H. et al. (2004), "Group social capital and group effectiveness: The role of informal socializing ties", en Academy of Management Journal , 47(6): 860-875.

Oh, H., Labianca, G. et al. (2006). "A multilevel model of group social capital", en Academy of Management Review, 31(3): 569-582.

Paasi, A. (2005), "Globalisation, academic capitalism, and the uneven geographies of international journal publishing spaces", en Environment and Planning, A 37: 769-789.

Perianes-Rodríguez, A.; Olmeda-Gómez, C. et al. (2010), "Detecting, identifying and visualizing research groups in co-authorship networks", en Scientometrics, 82(2): 307-319.

Reagans, R. y Zuckerman, E. W. (2001), "Networks, diversity, and productivity: The social capital of corporate $\mathrm{R} \& \mathrm{D}$ teams", en $\mathrm{Or}$ ganization Science, 12(4): 502.

Rey-Rocha, J., Martín-Sempere, M. J. et al. (2002), "Research productivity of scientists in consolidated vs. non-consolidated teams: The case of Spanish university geologists", en Scientometrics, 55(1): 137-156.

Rousseau, R. (1998), "Indicadores bibliométricos y econométricos en la evaluación de instituciones científicas", en Ci. Inf., Brasília, 27(2): $149-158$. 
Schummer, J. (2004), "Multidisciplinarity, interdisciplinarity, and patterns of research collaboration in nanoscience and nanotechnology", en Scientometrics, 59(3): 425-465.

Tichy, N. M., Tushman, M. L. et al. (1979), "Social network analysis for organizations", en The Academy of Management Review, 4(4): 507-519.

Tunzelmann, N.v.; Ranga, M. et al. (2003), The effects of size on research performance: A SPRU review, SPRU-Science and Technology Policy Research, University of Sussex.

Van-Raan, A. F. J. (2003), "The use of bibliometric analysis in research performance assessment and monitoring of interdisciplinary scientific developments", en Technology Assessment-Theory and Practice, 1(12): 20-29.

Wairepo, A. R. (2006), The Association between co-authorship network structures and successful academic publishing among higher education scholars, Department of Educational Leadership and Foundations, Brigham Young University, Doctor of Philosophy.

Walford, L. (2000), "The research assessment exercise: its effect on scholarly journal publishing", en Learned Publishing, 13: 49-52.

Wasserman, S. y Faust, K. (1994), Social network analysis, methods and applications, Cambridge: Cambridge University Press.

Wong, S. S. (2008), "Task knowledge overlap and knowledge variety: the role of advice network structures and impact on group effectiveness", en Journal of Organizational Behavior, 29: 591-614.

Yousefi-Nooraie, R.; Akbari-Kamrani, M. et al. (2008), "Association between co-authorship network and scientific productivity and impact indicators in academic medical research centers: A case study in Iran", en Health Research Policy and Systems, 6(9). 